\title{
Research Paper: Comparison Between Spatiotemporal Parameters and Vertical Ground Reaction Force in Ankle Sprain Coper and Healthy Athletes: A Cross Sectional Study
}

\author{
Zahra Raeisi ${ }^{* *}$ (D) \\ 1. Department of Sport Injuries and Corrective Exercises, Faculty of Sport Science, Arak University, Arak, Iran.
}

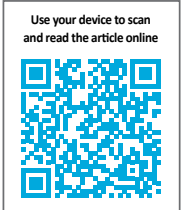

Citation Raeisi Z. Comparison Between Spatiotemporal Parameters and Vertical Ground Reaction Force in Ankle Sprain Coper and Healthy Athletes: A Cross Sectional Study. Physical Treatments. 2021; 11(2):111-120. http://dx.doi.org/10.32598/ ptj.11.2.474.1

: http://dx.doi.org/10.32598/ptj.11.2.474.1

\section{(i) (3)}

\section{Article info:}

Received: 18 Jul 2020

Accepted: 23 Jan 2021

Available Online: 01 Apr 2021

Keywords:

Ankle injury, Prevention, Joint instability, Gait analysis

\begin{abstract}
A B S T RA C T
Purpose: It is essential to maintain dynamic stability during walking to perform daily tasks independently. The present study aimed at comparing the spatiotemporal parameters and the values of the vertical Ground Reaction Force (vGRF) as well as determining the time to reach them in ankle-sprain coper and healthy athletes during the stance phase of gait.
\end{abstract}

Methods: A total of 28 female university athletes were recruited in this cross-sectional study and assigned into two groups: ankle-sprain coper $(n=14)$ and healthy control $(n=14)$. The gait cycle analysis was then performed on a $10-\mathrm{m}$ path, and the information related to the stance phase was recorded by a foot scanning device. The spatiotemporal parameters (gait line and contact time) and the values of the VGRF along with the time to reach them were subsequently obtained from each test. The repeated measures Analysis of Variance (ANOVA) was additionally used to analyze the data $(\mathrm{P} \leq 0.05)$.

Results: The study results revealed no differences between the injured and the healthy feet in the ankle-sprain coper group in any of the variables $(\mathrm{P}>0.05)$. As well, the spatiotemporal gait cycle parameters between the ankle-sprain coper group and the healthy controls were not significantly different $(\mathrm{P}>0.05)$. However, significant differences were observed between the ankle-sprain coper and healthy controls in terms of the variables of the vGRF in the mid-stance $(\mathrm{F}=5.25$, $\mathrm{P}=0.03)$ and the time to reach the second peak of the $\operatorname{vGRF}(\mathrm{F}=9.13, \mathrm{P}=0.006)$.

Conclusion: The spatiotemporal gait parameters were not significantly different between the ankle-sprain coper and the control groups, but the vGRF in the ankle-sprain coper was greater than that in the control group. With regard to the correlation between the reduction in the vGRF and the secondary injury, it is recommended to pay much attention to this point in rehabilitation programs following the first injury in female athletes.

\footnotetext{
* Corresponding Author:

Zahra Raeisi, PhD.

Address: Department of Sport Injuries and Corrective Exercises, Faculty of Sport Science, Arak University, Arak, Iran.

Phone: +98 (913) 3848636

E-mail: z_raisi13@yahoo.com
} 


\section{Highlights}

- The vGRF in the coper group was greater than the control group.

- The spatiotemporal parameters were not significantly different between groups.

- No differences observed in injured and healthy feet of coper group.

\section{Plain Language Summary}

Lateral Ankle Sprain (LAS) is the most common injury in people with physical activity. Chronic ankle instability is a pervasive term used to classify a person with mechanical and functional ankle instability. Among these, there are also people who do not experience recurrent ankle sprains after the initial injury, and are often referred to the "coper" group in studies. Walking is very important in daily life and its dynamic stability is essential for independence during daily activities. People with a history of LAS experience imbalance, increased lateral ankle pressure during stance phase of walking, and recurrent ankle sprain. Given the importance of ground reaction force and walking variables in the occurrence of injury, the purpose of this study was to compare spatiotemporal parameters of walking and vertical ground reaction force (VGRF) and time to vGRF values in the coper group and healthy control. 28 active female college students as coper ankle sprain $(\mathrm{n}=14)$ and healthy control $(\mathrm{n}=14)$ groups participated in the present study. The gait-test was performed on a 10-meter path and the information related to the stance phase was recorded by the foot scanning device. The spatiotemporal parameters and the values of the vGRF and time to reach them were obtained from each test. The results showed that the coper group did not differ from the healthy control group in spatiotemporal parameters. Unlike spatiotemporal parameters, the vGRF in the coper group was higher than in the control, which it can be the cause cartilage of the ankle joint damages. Due to the relationship between vGRF value and secondary injury reduction, training of right walking as the main part of rehabilitation programs can be one of the therapeutic goals to prevent the frequency of ankle sprain and re-injury in coper athletes.

\section{Introduction}

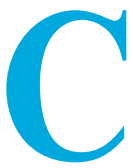

hronic Ankle Instability (CAI) is a pervasive term utilized to classify a person with mechanical and functional ankle instability. In this respect, residual symptoms such as repeated episodes of giving-way and ankle instability should be present for at least one year following the first sprain, so that affected individuals can be classified as cases with CAI [1-3]. However, some individuals do not experience such recurrent ankle sprains after primary injuries and are often referred to as "ankle-sprain coper" groups in different studies. Regarding a review study by Wikstrom and Brown, three key components need to be included in the ankle-sprain coper operational definitions: 1) the first Lateral Ankle Sprain (LAS), 2) absence of CAI symptoms such as no giving way or suffering from ankle disability, and 3) time component [2]. In another study, Wikstrom et al. had found that ankle-sprain coper groups had no experience of recurrent ankle sprains or episodes of giving way, and they could even return to the minimum average level of physical activity with no restrictions after at least 12 months [3].
Walking is essential in daily life, and its dynamic stability is essential to perform everyday activities independently $[4,5]$. People with a history of LAS might thus experience imbalance, increased lateral ankle pressures during the gait cycle stance phase, as well as repeated occurrence of ankle sprains [6] Moreover, it has been suggested that a large number of ankle sprains appear as a result of maximum supination torque in the subtalar joint, often due to the extent and the position of the vertical Ground Reaction Force (vGRF) at the onset of the contact time of the foot [7]. If unbalanced mechanical loads are correspondingly applied to the talus at a high rate for a long time, the structural properties of the articular cartilage (such as collagen, proteoglycan, and water contents) may be affected, and this can be the leading cause of destructive ankle osteoarthritis [8].

The results of studies in this field have similarly demonstrated that patients with LAS reluctantly put their weight on the front third [5]. Increased changes in foot and ankle movements may further multiply the risks of recurrent ankle sprains, even if there is no mechanical damage to the ligament. Such changes are consistent with the LAS tolerance mechanism [9]. The researchers 
evaluated postural control, sensorimotor, perceptual, and mechanical differences between copers and CAI during similar landing and cutting maneuvers [2, 10-13]. Their results showed that patients with CAI had more changes in landing mechanics that may predispose them to recurrent LAS. While comparing coper and healthy controls showed lees change.

Moreover, the findings of Son et al. showed a $2.5^{\circ} \mathrm{C}$ reduction in dorsiflexion angle in copers during mid-landing compared to the healthy control. As a result, the researchers have suggested that reducing the dorsiflexion range of motion increases the risk of ankle sprain injury to five times [14]. On the other hand, reducing movement changes can be one of the main goals of preventive strategies targeting repeated ankle sprains [15]. In this regard, Brown et al. had compared CAI and ankle-sprain coper groups and concluded that individuals with functional ankle instability had shown significant changes in their ankles on the frontal plane compared with the control and ankle-sprain coper groups [10]. Modified movement strategies evolved after ankle sprain as a post-injury adaptation. According to various studies, the researchers stated that copers use good movement strategies to prevent recurrent LAS in activities such as landing and cutting. Wikstrom and Hass determined the biomechanical control alterations present in copers, controls, and those with chronic ankle instability during planned and unplanned gait termination. Their results showed a change in muscle activity at the end of the gait phase, indicating a reduction in existing strategies in patients with CAI, while copers at the gait termination use a method similar to uninjured control [16]. Of note, delayed or inadequate reactions to environmental changes may also impair the ability of injured people to maintain the gait cycle balance and may accordingly account for the tendency to recurrent ankle sprains as well as persistent symptoms [17].

In this sense, the coordinated and integrated functioning of various human body organs is vital for continuous walking with no falls. In the meantime, the lower limbs play vital roles in establishing this consistent pattern through applying the force of gravity resulting from reaching the ground, maintaining balance, and creating forward forces [15]. The vGRF and the time to reach the vGRF values are thus associated with lower limb injuries and ankle sprains. Given the high prevalence rates of ankle sprains and their consequences, high probability of recurrent ankle sprains together with high costs of medical healthcare, the prevention of secondary sprains is essential in the event of primary injuries. Despite numerous studies on walking in CAI patients, little research has reflected on ankle-sprain coper groups. Considering the importance of the Ground Reaction Force (GRF) and walking variables in the occurrence of injuries, this study aimed to compare the spatiotemporal gait cycle parameters and the vGRF and determine the time to reach the vGRF values in ankle-sprain coper athletes and healthy controls. The present study results can help determine the underlying risk factors for recurrent LAS by recognizing the differences between ankle-sprain coper and healthy control athletes and help identify the factors that are more likely to LAS following an initial ankle sprain injury.

\section{Materials and Methods}

A total of 28 female colleague athletes, with demographic characteristics listed in Table 1, were recruited in this cross-sectional study in the form of two groups: ankle-sprain coper and healthy control. The sample size was also estimated at 14 using the $\mathrm{G}^{*}$ Power software for each group, considering the 0.8 test power, 0.05 significance level, and 0.6 effect size [18]. The inclusion criteria for both groups involved the age range of 18-25 years, no history of neurological diseases as well as hearing and vision problems, leg length inequality, and fractures or surgeries on the trunk and the lower limbs in the six months leading up to the study, along with regular physical activity at least three sessions a week for the last six months before the research $[2,19]$. The dominant leg in all the athletes in both groups was the right one, determined through the ball-kicking test. In this sense, the inclusion criteria for the ankle-sprain coper group were a history of LAS (only once) leading to numbness or inability to bear weight for three days after injury, at least 12 months after the first ankle sprain, and a return to physical activity before the injury, recurrent ankle sprains, a score of 25-28 on the Cumberland Ankle Instability Tool (CAIT), episodes of giving way or disability in the ankle, and history of sprains in the dominant leg $[2,19]$.

On the other hand, the inclusion criteria for the healthy controls embraced no history of ankle sprains, homogeneity in terms of the dominant leg, height, and weight compared with the ankle-sprain coper group, and a score of $>29$ on the CAIT [19]. If the subjects were unwilling to complete the tests or discontinue, they could withdraw at any stage of the study. It is noteworthy that the present study received approval from the Research Ethics Committee of Bu-Ali Sina University, Hamedan (Code: IR.BASU.REC.1398.015).

At the first stage, once the subjects entered the sports medicine research and testing laboratory at Arak University, the study objectives and protocols were explained 
by the tester. Upon obtaining their consent to be included in the study, the subjects also filled out the forms related to the demographic characteristics and then completed the CAIT. It is one of the valid and reliable questionnaires designed explicitly for ankle instability and used to classify people with ankle instability [20, 21]. Mirshahi et al. assessed the reliability of the Persian version of the CAIT in Iranian athletes affected with ankle sprains. The researchers had concluded that the Persian version could be administered as a reliable tool to diagnose ankle instability in athletes [22]. Hiller first published this questionnaire in 2006. It comprised 9 items involving 30 points to identify and examine the severity of functional ankle instability. Accordingly, 8 out of 9 items had been designed to assess ankle instability in subjects through their exercise or daily activities, and one item had been intended to determine the time subjects might feel pain [23]. The CAIT scores ranged from 0 to 30, with a higher score indicating a higher degree of ankle stability and a lower one denoting ankle instability [24].

In the present study, the spatiotemporal parameters (gait line and contact time) and the vGRF values, as well as the time to reach them in the gait cycle stance phase, were recorded by a plantar scanner (manufactured by Dirce, Germany). This scanner was equipped with 4096 sensors, each with the dimensions of $5 \times 7 \mathrm{~mm}^{2}$ and the active sensor area of $480 \times 320 \mathrm{~mm}$ in total. The given system also had a sensitivity of $0.72-127 \mathrm{~N} / \mathrm{cm}^{2}$ and a sampling frequency of $300 \mathrm{~Hz}$.

After completing the questionnaires, the demographic variables were measured. The subjects were then asked to perform the $10-\mathrm{m}$ walk test, with a force plate in the middle, at the desired speed and barefoot. Initially, each subject was allowed five times to practice and was asked to walk the path while looking forward. After getting acquainted with the task and the path, the tests were performed, and three correct repetitions were recorded for each foot from each subject. The recorded data on the GRF were additionally filtered using the low-pass fourth-order Butterworth filter with no fuzzy difference at a cutoff frequency of $20 \mathrm{~Hz}$. Then, the measurements included the Peak vGRF (PvGRF) at the initial contact time (the first peak of the vertical force, FzI.C) and the time to reach it (TzI.C), the vGRF value in the midstance phase of walking (minimum force, FzM.S) and the time to reach it (TzM.S), and finally the PvGRF at the push-off phase (i.e., the second peak of the vertical force, FzP.O) and the time to reach it (TzP.O). The data on the vGRF for normalization was then divided by the weight of the subjects (Newton).
The data were ultimately elucidated via descriptive statistics, mean, and standard deviation. The inferential statistics were also recruited. They included the Shapiro-Wilk test to examine the normality of data distribution, the independent samples t-test to find the difference between demographic characteristics of the subjects, and the repeated measures Analysis of Variance (ANOVA) to calculate the intra-group and inter-group results of the parameters related to walking at the significance level of $\mathrm{P} \leq 0.05$.

\section{Results}

The demographic characteristics, including age, height, weight, Body Mass Index (BMI), a history of ankle sprains, and the CAIT scores in the study groups are presented in Table 1. In this respect, the independent samples t-test results showed that the groups did not differ significantly in terms of the demographic variables $(\mathrm{P}>0.05)$. However, there was a significant difference between the study groups in the CAIT scores $(\mathrm{t}=11.25, \mathrm{P}<0.001)$

Besides, the results of the intra-group comparisons revealed no significant difference between the two feet in the ankle-sprain coper athletes group in the variables of the contact time of the injured and healthy feet $(\mathrm{F}=0.15, \mathrm{P}=0.69)$ and the Center of Pressure $(\mathrm{CoP})$ gait line $(\mathrm{F}=1.1, \mathrm{P}=0.3)$. Moreover, no significant difference was observed in the contact time of the injured foot between the ankle-sprain coper athletes group and its corresponding foot in the control group $(\mathrm{F}=1.63, \mathrm{P}=0.21)$ (Tables 2 and 3 ) according to the results obtained from the inter-group comparisons. Besides, there was no significant difference in the walking gait line between the ankle-sprain coper and control groups $(\mathrm{F}=1.47, \mathrm{P}=0.23)$ (Tables 2 and 4).

The results of the statistical analyses confirmed no significant difference in the values of the first peak of the vGRF $(\mathrm{F}=3.87, \mathrm{P}=0.06)$, the minimum force of the vGRF $(\mathrm{F}=1.4, \mathrm{P}=0.24)$, and the second peak of the vGRF ( $\mathrm{F}=0.1, \mathrm{P}=0.75)$ between the injured and healthy feet in the ankle-sprain coper group (Figure 1) (Table 3).

As depicted in Figure 1 and Table 3, there was no remarkable difference in the values of the time reach to the first PvGRF ( $\mathrm{F}=2.01, \mathrm{P}=0.17$ ), the time to reach the minimum force of the vGRF $(\mathrm{F}=2, \mathrm{P}=0.17)$, and the time to reach the second PvGRF $(\mathrm{F}=1.95, \mathrm{P}=0.176)$ between the injured and healthy feet in the ankle-sprain coper group.

Moreover, statistical analyses demonstrated no significant difference in the values of the first PvGRF ( $\mathrm{F}=2.14$, $\mathrm{P}=0.15)$ and the second $\mathrm{PvGRF}(\mathrm{F}=2.2, \mathrm{P}=0.15)$ between 
Table 1. Mean \pm SD of demographic characteristics of study subjects

\begin{tabular}{|c|c|c|c|c|}
\hline Variables & Coper Group & Control Group & $\mathbf{t}$ & $\mathbf{P}$ \\
\hline Height (cm) & $166.8 \pm 2.59$ & $165.7 \pm 2.45$ & 1.12 & 0.27 \\
\hline Weight (kg) & $54.7 \pm 2.0$ & $55.2 \pm 2.3$ & 0.52 & 0.6 \\
\hline Body mass index $\left(\mathrm{kg} / \mathrm{m}^{2}\right)$ & $19.6 \pm 1.05$ & $19.89 \pm 0.78$ & 0.54 & 0.59 \\
\hline CAIT score ${ }^{a}$ & $26.2 \pm 0.9$ & $29.7 \pm 0.46$ & 11.25 & $0.001^{*}$ \\
\hline Ankle sprain history & 1 & 0 & - & \\
\hline
\end{tabular}

${ }^{a}$ Cumberland Ankle Instability Tool score

PHYSICAL TREA IMENTS

${ }^{*} \mathrm{P} \leq 0.05$

Table 2. Mean $\pm S D$ of spatiotemporal parameters in walking stance phase

\begin{tabular}{cccc}
\hline \multirow{2}{*}{ Groups } & Limb & \multicolumn{2}{c}{ Mean \pm SD } \\
& & Gait Line (mm) & Contact Time (s) \\
\hline \multirow{2}{*}{ Coper } & Injured foot & $206.19 \pm 13.73$ & $0.68 \pm 0.04$ \\
& Uninjured foot & $207.2 \pm 9.6$ & $0.68 \pm 0.03$ \\
\hline \multirow{2}{*}{ Control } & Dominant foot (corresponding to coper group injured foot) & $213.13 \pm 11.87$ & $0.66 \pm 0.04$ \\
\hline & & & PHYSICAL TREA MENTS
\end{tabular}

the injured foot of the ankle-sprain coper group and its corresponding foot in the healthy control athletes. Similarly, the results revealed a significant difference in the minimum force of the PvGRF ( $\mathrm{F}=5.25, \mathrm{P}=0.03$ ) between the injured foot of the ankle-sprain coper group and its corresponding foot in the healthy controls (Figure 2) (Table 4).
As observed in Figure 2 and Table 4, there was no significant difference between the injured foot in the anklesprain coper group and its corresponding foot in the healthy control athletes in the values of the time to reach the first PvGRF ( $\mathrm{F}=0.96, \mathrm{P}=0.33)$ and the time to reach the minimum force of the vGRF $(\mathrm{F}=1.23, \mathrm{P}=0.27)$. Accordingly, the results proved a significant difference between the injured foot of the ankle-sprain coper group and

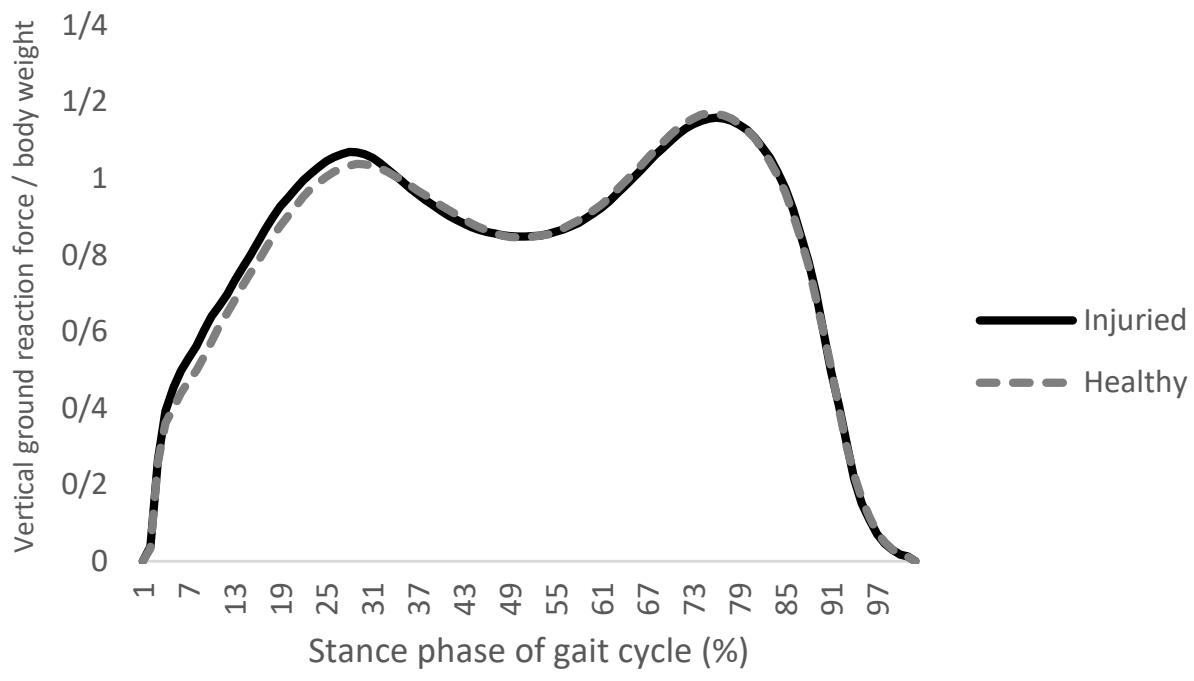

PHYSICAL TREA M MENTS

Figure 1. Vertical ground reaction force in injured and healthy feet of coper group based on the percentage of the stance phase of gait 


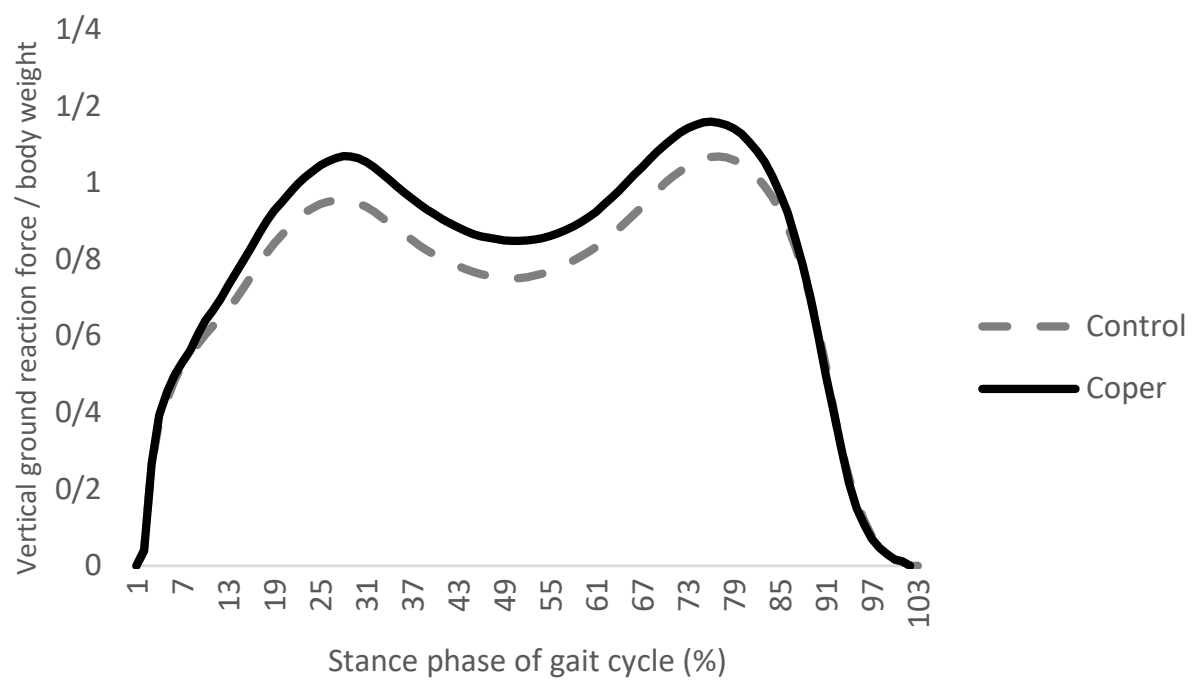

PHYSICAL TREATMENTS

Figure 2. Vertical ground reaction force in the injured foot of the coper group and its corresponding foot in the healthy control athletes based on the percentage of the stance phase of gait

its corresponding foot in the healthy control athletes one during the walking stance phase in the time to reach the second PvGRF (F=9.13, $\mathrm{P}=0.006$ ) (Figure 2) (Table 4).

\section{Discussion}

This study aimed to compare the spatiotemporal gait cycle parameters and the vGRF values in active anklesprain coper and healthy control athletes. The results demonstrated no significant difference between the injured and healthy feet in any of the variables examined in the ankle-sprain coper group. The spatiotemporal parameters also revealed no difference between the injured foot in the ankle-sprain coper group and its corresponding foot in the healthy athletes. The PvGRF values in the mid-stance phase of walking (minimum force) and the time to reach the second peak of vertical force (FzP.O) in the ankle-sprain coper group similarly established a significant difference compared with the control group.
It should be noted that neuromuscular problems, musculoskeletal injuries, or any other disorders can cause changes in the correct pattern of walking in different directions, which may affect the structure of the limbs, especially the lower ones, in the long run [25]. In this sense, individuals with LAS and CAI also have difficulty walking, controlling their posture, and changing motor strategies while walking [26]. Although there are changes in the gait cycle for days and weeks after acute LAS, long-term changes in the gait in ankle-sprain coper groups are not fully understood [27].

Evidence suggests that ankle-sprain coper athletes may have different patterns of movement than CAI individuals and control groups in various tasks and actions [8]. On the other hand, the presence of inversion has been observed more in the back foot of patients with subacute LAS during the mid-stance phase compared with the healthy control group. In this sense, the present study results on the spatiotemporal parameters indicated no difference between the ankle-sprain coper group and the

Table 3. Repeated measure ANOVA for the injured and healthy feet in the ankle-sprain coper group (intra-group analysis)

\begin{tabular}{ccccccccc}
\hline Result & Fzl.C & FzM.S & FzP.O & Tzl.C & TzM.S & TzP.O & CT & GL \\
\hline F & 3.87 & 1.4 & 0.1 & 2.01 & 2 & 1.95 & 0.15 \\
p & 0.06 & 0.24 & 0.75 & 0.17 & 0.17 & 0.176 & 0.69 \\
\hline
\end{tabular}

FzI.C, The first peak of the vertical ground reaction force; FzM.S, The minimum of the vertical ground reaction force at the midstance phase; FzP.O, The peak of the vertical ground reaction force at the push-off phase; TzI.C, The time to reach the first peak of the vertical ground reaction force; TzM.S, The time to reach the minimum of the vertical ground reaction force at the mid-stance phase; TzP.O, The time to reach the peak of the vertical ground reaction force at the push-off phase; CT, Contact time; GL, Gait line. 
Table 4. Repeated measure ANOVA for the injured foot of the coper group and its corresponding foot in the healthy control athletes (inter-group analysis)

\begin{tabular}{cccccccrr}
\hline Result & FzI.C & FzM.S & FzP.O & TzI.C & TzM.S & TzP.O & Contact Time & Gait Line \\
\hline F & 2.14 & 5.25 & 2.2 & 0.96 & 1.23 & 9.13 & 1.63 \\
p & 0.15 & $0.03^{*}$ & 0.15 & 0.33 & 0.27 & $0.006^{*}$ & 0.21 & 0.23 \\
\hline
\end{tabular}

FzI.C, The first peak of the vertical ground reaction force; FzM.S, The minimum of the vertical ground reaction force at the mid-stance phase; FzP.O, The peak of the vertical ground reaction force at the push-off phase; TzI.C, The time to reach the first peak of the vertical ground reaction force; TzM.S, The time to reach the minimum of the vertical ground reaction force at the mid-stance phase; TzP.O, The time to reach the peak of the vertical ground reaction force at the push-off phase.

*P $\leq 0.05$; pairs of groups significantly

healthy controls and the injured and healthy feet in the ankle-sprain coper group [10]. Accordingly, decreased gait changes might be due to a tendency to be conservative, and on the other hand, augmented variability may indicate the flexibility of the Central Nervous System (CNS) and the adaptability to the gait cycle changes while walking [10]. Because the changes indicated the CNS control over gait [17], these findings could imply different compatibility patterns of the CNS in the anklesprain coper group.

Based on the results of previous studies on the gait cycle, the CAI individuals had shown lower walking speed, lower number of steps short in length, wider support surface, and more variable movement patterns compared with the control group. These results suggest that the biological system is seeking optimal solutions to produce walking stability $[9,27]$. The incidence of LAS in individuals could further cause a decrease in walking speed, step length, and single-support time, but an increase in plantar flexion, as well as heel inversion before and after initial contacts, compared with healthy groups. Concerning the study results and the lack of differences in the spatiotemporal parameters between the ankle-sprain coper and control groups, the ankle-sprain coper group may successfully compensate for the altered patterns generated probably by LAS, whereas this compensation has not occurred in people with CAI. This similarity to the healthy athletes could be one reason for the reduced risk of inversion ankle sprain in the anklesprain coper athletes.

It should be noted that walking is one of the essential activities in daily life. Among the various parts of the lower limb, the role of the ankle as the closest joint to the source of contact energy during contact with the ground may be considered more critical. Therefore, contact forces are absorbed during movements in this joint [1]. The GRF is not the only force acting on the joints when walk- ing, but the weight and the immobility of a moving part can affect the adjacent distal and proximal parts. Following the contact of the foot with the ground, the vGRF is transmitted to the ankle through the fat planes located in the heel and talus and then through the ankle to the movement chain, which is eventually removed by the joints. If the range of motion is reduced due to the body's efforts to protect the ankle, this may lead to a growth in peak forces [28]. In voluntary movement patterns, the GRF is also produced as two peak points and one depth point between the two peaks. In this sense, the first peak force is produced simultaneously with the contact of the limb with the surface and until the beginning of the contact phase with the ground, where the limb receives the weight of the body and the depth force simultaneously with the full plantar contacting the ground. Ultimately, the second peak force is generated by the toe pressure on the ground to produce the push-off force [25, 29].

The present study results confirmed an increase in the vGRF in the ankle-sprain coper group compared to the healthy athletes. A significant difference was additionally observed in the GRF value during the mid-stance phase (minimum force). Besides, the ankle-sprain coper group showed a growth in the minimum force during the stance phase. The time to reach the second peak of the vGRF in the ankle-sprain coper group was significantly faster than that in the controls, suggesting that the joint was subjected to more loads in a shorter time. The maximum reaction force timing was also consistent with the ankle vulnerability because the LAS could occur shortly after the peak force in most cases. Therefore, the presence of ankle-sprain coper in this period, which was more vulnerable, would intensify the risk of injuries [30].

According to Fraser et al., the CAI group had shown higher-impact peak forces than the control group. Likewise, the CAI group had a higher loading rate and a shorter time to reach the active peak force than the control group [26]. As 
stated by Bigouette et al., people with CAI had demonstrated a growing trend in the vGRF and the loading rate and a shorter time to reach the active peak force than the control group [28]. Although most previous studies had evaluated people with CAI, Brown et al. reflected on the active peak vGRF and the time to reach the peak vGRF while running in the group with functional ankle instability and ankle-sprain coper. For the PvGRF, they had not found significant differences between the two sub-groups of CAI (mechanical and functional ankle instability) compared with the ankle-sprain coper group [10].

Wikstrom and Hass, in comparison with copers and CAI, also reported that people with CAI showed greater peak braking forces and relied more on the braking limb, while copers could reduce the propulsion force on the lead limb and increase the braking force on the brake limb which showed a pattern similar to that of uninjured control [16]. The researchers speculated that these results might indicate a coping mechanism in copers that does not exist in CAI. However, they believed the results needed further research [16]. An increase in the vGRF values during the stance phase in the present study, which was observed in the ankle-sprain coper group, may indicate incompatibility strategies expanded after the first sprain or the injured pattern of gait and may be a reason to expose these individuals to the first injury. One of the limitations of the present study was to evaluate the GRF only in the vertical direction. In this study, only the walking stance phase was examined. The subjects recruited in the present study were female athletes, so studies on male cases may provide different findings.

\section{Conclusion}

The present study results suggest no differences between the foot with previous sprains and the healthy one in the ankle-sprain coper group. Moreover, this group did not differ from the healthy controls regarding the spatiotemporal gait cycle parameters. Unlike the spatiotemporal parameters, the vGRF in the ankle-sprain coper group was higher than that in the controls, which could jeopardize the long-term health of the articular cartilage of the ankle. Concerning the relationship between reducing the vGRF and lower secondary injuries, proper gait training in rehabilitation programs can be one of the therapeutic and preventive goals targeting recurrent ankle sprains in ankle-sprain coper athletes. Further research is also needed to compare ankle-sprain coper individuals with healthy controls.

\section{Ethical Considerations}

\section{Compliance with ethical guidelines}

The present study received approval from the Research Ethics Committee of Bu-Ali Sina University, Hamedan, Iran (Code: IR.BASU.REC.1398.015).

\section{Funding}

This research was supported by Arak University, Arak City, Iran (Grant no.: 97/15013).

\section{Conflict of interest}

The author declared no conflict of interest.

\section{References}

[1] Delahunte E, Coughlan GF, Caulfield B, Nightingale EJ, Christine Lin CW, Hiller CE. Inclusion criteria when investigating insufficiencies in chronic ankle instability. Medicine and Science in Sports and Exercise. 2010; 42(11):2106-21 [DOI:10.1249/MSS.0b013e3181de7a8a] [PMID]

[2] Wikstrom EA, Brown CN. Minimum reporting standards for copers in chronic ankle instability research. Sports Medicine. 2014; 44:251-68. [DOI:10.1007/s40279-013-0111-4] [PMID]

[3] Wikstrom EA, Tillman MD, Chmielewski TL, Cauraugh JH, Naugle KE, Borsa PA. Discriminating between copers and people with chronic ankle instability. Journal of Athletic Training. 2012; 47(2):136-42. [DOI:10.4085/1062-605047.2.136] [PMID] [PMCID]

[4] Northeast L, Gautrey CN, Bottoms L, Hughes G, Mitchell ACS, Greenhalgh A. Full gait cycle analysis of lower limb and trunk kinematics and muscle activations during walking in participants with and without ankle instability. Gait \& Posture. 2018; 64:114-8. [DOI:10.1016/j.gaitpost.2018.06.001] [PMID]

[5] Mahmood I, Martinez-Hernandez U, Dehghani-Sanij AA. Evaluation of gait transitional phases using neuromechanical outputs and somatosensory inputs in an overground walk. Human Movement Science. 2020; 69:102558. [DOI:10.1016/j.humov.2019.102558] [PMID]

[6] Koldenhoven RM, Feger MA, Fraser JJ, Hertel J. Variability in center of pressure position and muscle activation during walking with chronic ankle instability. Journal of Electromyography and Kinesiololy. 2018; 38:155-61. [DOI:10.1016/j. jelekin.2017.12.003] [PMID]

[7] Fong DT, Chan YY, Mok KM, Yung PS, Chan KM. Understanding acute ankle ligamentous sprain injury in sports. BMC Sports Science Medicine and Rehabilitation. 2009; 1:14 [DOI:10.1186/1758-2555-1-14] [PMID] [PMCID]

[8] Jun Son S, Kim H, Seeley MK, Ty Hopkins J. Altered walking neuromechanics in patients with chronic ankle in- 
stability. Journal of Athletic Traininig. 2019; 54(6):684-97. [DOI:10.4085/1062-6050-478-17] [PMID] [PMCID]

[9] Torp DM, Thomas AC, Donovan L. External feedback during walking improves measures of plantar pressure in individuals with chronic ankle instability. Gait \& Posture. 2019; 67:236-41. [DOI:10.1016/j.gaitpost.2018.10.023] [PMID]

[10] Brown C, Padua D, Marshall SW, Guskiewicz K. Individuals with mechanical ankle instability exhibit different motion patterns than those with functional ankle instability and ankle sprain copers. Clinical Biomechanics. 2008; 23(6):822-31. [DOI:10.1016/j.clinbiomech.2008.02.013] [PMID]

[11] Shah S, Thomas AC, Noone JM, Blanchette CM, Wikstrom EA. Incidence and cost of ankle sprains in United States emergency departments. Sports Health. 2016; 8(6):547-52. [DOI:10.1177/1941738116659639] [PMID] [PMCID]

[12] Sefton JM, Hicks-Little CA, Hubbard TJ, Clemens MG, Yengo CM, Koceja DM, et al. Sensorimotor function as a predictor of chronic ankle instability. Clinical Biomechanics. 2009; 24(5):451-8. [DOI:10.1016/j.clinbiomech.2009.03.003] [PMID]

[13] Hubbard TJ. Ligament laxity following inversion injury with and without chronic ankle instability. Foot \& Ankle International. 2008; 29(3):305-11. [DOI:10.3113/FAI.2008.0305] [PMID]

[14] Son SJ, Kim H, Seeley MK, Hopkins JT. Movement strategies among groups of chronic ankle instability, coper, and control. Medicine \& Science Sports \& Exercise. 2017; 49(8):164961. [DOI:10.1249/MSS.0000000000001255] [PMID]

[15] Jamali A, Forghany S, Bapirzadeh K, Nester C. The effect of three different insoles on ankle movement variability during walking in athletes with functional ankle instability. Advanced Biomedical Research. 2019; 8:42. [DOI:10.4103/abr. abr_69_19] [PMID] [PMCID]

[16] Wikstrom EA, Hass CJ. Gait termination strategies differ between those with and without ankle instability. Clinical Biomechanics. 2012; 27(6):619-24. [DOI:10.1016/j.clinbiomech.2012.01.001] [PMID]

[17] Springer S, Gottlieb U. Effects of dual-task and walking speed on gait variability in people with chronic ankle instability: A cross-sectional study. BMC Musculoskeletal Disorders. 2017; 18:316. [DOI:10.1186/s12891-017-1675-1] [PMID] [PMCID]

[18] Kadam P, Bhalerao S. Sample size calculation. International Journal of Ayurveda Research. 2010; 1(1):55-7. [DOI:10.4103/0974-7788.59946] [PMID] [PMCID]

[19] Lin JZ, Lin YA, Lee HJ. Are landing biomechanics altered in elite athletes with chronic ankle instability. Journal of Sports Science \& Medicine. 2019; 18(4):653-62. https:/ /www.jssm. org/jssm-18-653.xml\%3EFulltext

[20] Hiller CE, Refshauge KM, Bundy AC, Herbert RD, Kilbreath SL. The cumberland anklei instability tool: A report of validity and reliability testing. Archives of Physical Medicine and Rehabilitation. 2006; 87(9):1235-41. [DOI:10.1016/j. apmr.2006.05.022] [PMID]

[21] Wright CJ, Arnold BL, Ross SE, Linens SW. Recalibration and validation of the Cumberland ankle instability tool cutoff score for individuals with chronic ankle instability. Archives of Physical Medicine and Rehabilitation. 2014; 95(10):1853-9. [DOI:10.1016/j.apmr.2014.04.017] [PMID]
[22] Mirshahi M, Halabchi F, Golbakhsh MR, Saadat S. Reliability and recalibration of the Persian version of cumberland ankle instability tool cut-off score in athletes with functional ankle instability. Advanced Journal of Emergency Medicine. 2019;3(3):e26. https://fem.tums.ac.ir/index.php/fem/article/view/180

[23] Martin RL, Davenport TE, Paulseth S, Wukich DK, Godges JJ, Altman MR, et al. Ankle stability and movement coordination impairments: Ankle ligament sprains. Journal of Orthopaedic \& Sports Physical Therapy; 2013; 43(9):A1-40. [DOI:10.2519/jospt.2013.0305] [PMID]

[24] Henderson EM. Evaluation of the cumberland ankle instability tool as a predictor of ankle re-injury in collegiate athletes [MA thesis]. Newark: University of Delaware; 2015. http://udspace.udel.edu/handle/19716/17282

[25] Sadeghi H, Mousavi SK, Ghasempur H, Nabavinik H. [A comparison of the vertical ground reaction force during forward and backward walking in athletes with ankle sprain (Persian)]. Journal of Modern Rehabilitation. 2013; 7(1):7-12. https://mrj.tums.ac.ir/article-1-2-en.html

[26] Fraser JJ, Hart JM, Saliba SF, Park JS, Tumperi M, Hertel J. Multisegmented ankle-foot kinematics during gait initiation in ankle sprains and chronic ankle instability. Clinical Biomechanics. 2019; 68:80-8. [DOI:10.1016/j.clinbiomech.2019.05.017] [PMID]

[27] Krishan K, Kanchan T, Passi N. Estimation of stature from the foot and its segments in a sub-adult female population of North India. Journal of Foot and Ankle Research. 2011; 4(1):24. [DOI:10.1186/1757-1146-4-24] [PMID] [PMCID]

[28] Bigouette J, Simon J, Liu K, Docherty CL. Altered vertical ground reaction forces in participants with chronic ankle instability while running. Journal of Athletic Training. 2016; 51(9):682-7. [DOI:10.4085/1062-6050-51.11.11] [PMID] [PMCID]

[29] Yalfani A, Maleki B, Raeisi Z. [The effect of aquatic exercise therapy on the pain, disability and gait parameters of women with chronic low back pain (Persian)]. Research in Sort Medicine and Technology. 2019; 17(18):57-67. https:/ / jsmt.khu.ac.ir/article-1-401-en.html

[30] Yousefi M, Sadeghi H. [Ankle movement pattern variation during walking in people with functional ankle instability: A review article (Persian)]. The Scientific Journal of Rehabilitation Medicine, 2017; 6(2):234-45. [DOI:10.22037/ jrm.2017.1100330] 
This Page Intentionally Left Blank 